\title{
Suplementação com arginina na terapia nutricional de pacientes com câncer de cabeça e pescoço
}

\author{
Arginine supplementation in nutritional therapy of patients with head and neck cancer
}

Suplementación con arginina en la terapia nutricional de pacientes con cáncer de cabeza y cuello

Chrystiano de Campos Ferreira ${ }^{1,2 *}$, Wudson Henrique Alves de Araújo², Talita Bezerra Silva², Ana Luíza Neves de Assis², Rafael Horácio de Brito ${ }^{1}$, Jacksone Pena Feliciano', Potthyer Vieira Rocha1, Herika Rangel Ferreira ${ }^{3}$, Rodolfo Korte ${ }^{1,2}$.

\section{RESUMO}

Objetivo: Realizar uma revisão sistemática do efeito da suplementação da arginina em pacientes com câncer de cabeça e pescoço. Métodos: A seleção de artigos foi realizada por dois revisores independentes sendo revistas por um terceiro revisor no período de julho a agosto de 2018. A busca foi realizada nas bases de dados PubMed, CENTRAL e Lilacs. Resultados: Foram encontrados um total de 108 artigos, destes 96foram eliminados por não atenderem aos nossos critérios de inclusão. Assim chegou-se a um total de 12artigos utilizados nesta revisão. Considerações finais: Concluímos com o presente estudo que a suplementação com arginina nestes pacientes promoveu uma diminuição no índice de fístulas pós-operatórias nos pacientes que fizeram uso da suplementação com arginina em relação aos que fizeram uso da dieta padrão no período pós-operatório.

Palavras-chave: Neoplasias de cabeça e pescoço, Arginina, Nutrição oncológica.

\begin{abstract}
Objective: To perform a systematic review of the effect of arginine supplementation in patients with head and neck cancer. Methods: The selection of articles was performed by two independent reviewers being reviewed by a third reviewer from July to August 2018. The search was performed in PubMed, CENTRAL and Lilacs databases. Results: A total of 108 articles were found, of which 96 were eliminated because they did not meet our inclusion criteria. Thus, a total of 12 articles were used in this review. Final considerations: We conclude with the present study that arginine supplementation in these patients promoted a decrease in the index of postoperative fistulas in patients who used arginine supplementation in relation to those who used the standard diet in the postoperative period.
\end{abstract}

Key words: Head and neck neoplasms, Arginine, Oncological nutrition.

\section{RESUMEN}

Objetivo: Realizar una revisión sistemática del efecto de la suplementación de la arginina en pacientes con cáncer de cabeza y cuello. Métodos: La selección de artículos fue realizada por dos revisores independientes siendo revisados por un tercer revisor en el período de julio a agosto de 2018. La búsqueda fue realizada en las bases de datos PubMed, CENTRAL y Lilacs. Resultados: Se encontraron un total de 108 artículos, de

\footnotetext{
${ }^{1}$ Hospital de câncer de Barretos - Hospital de Amor Amazônia, Porto Velho-RO. *E-mail:drchrystianoferreira@gmail.com

2 Universidade Federal de Rondônia (UNIR), Porto Velho-RO.

${ }^{3}$ Secretaria de Saúde do Estado de Rondônia, Porto Velho-RO.
} 
estos 96 fueron eliminados por no atender a nuestros criterios de inclusión. Así se llegó a un total de 12 artículos utilizados en esta revisión. Consideraciones finales: Concluimos con el presente estudio que la suplementación con arginina en estos pacientes promovió una disminución en el índice de fístulas postoperatorias en los pacientes que hicieron uso de la suplementación con arginina en relación a los que hicieron uso de la dieta estándar en el período postoperatorio.

Palabras clave: Neoplasias de cabeza y cuello, Arginina, Nutrición oncológica.

\section{INTRODUÇÃO}

Segundo Galbiatti ALS, et al. (2013), o câncer de cabeça e pescoço é um dos tipos de neoplasias mais comuns em todo o mundo. Estima-se para o Brasil 10,86 novos casos de câncer de cavidade oral a cada 100 mil homens e de 3,28 para cada 100 mil mulheres. Assim é o quinto mais comum entre homens e o $12^{\circ}$ nas mulheres (INCA, 2017).

Nos pacientes com câncer de cabeça e pescoço a perda de peso é notória e ocorre devido à própria doença, que evolui, muitas vezes, com obstrução da via alimentar pelo tumor. Além disso, há associação da consumpção pelo processo patológico neoplásico como pelos tratamentos quimio e radioterápicos, que tendem a agravar o caso (SILVA MPN, et al., 2006). Assim, os tratamentos de pacientes com câncer de cabeça e pescoço associam-se a maiores taxas de imunossupressão e desnutrição, predispondo o paciente a maiores incidências de infecções (FRUCHTEBICHT AVG, et al., 2018; AGGARWAL BB, et al., 2009).

Diante da complexidade de nutrição dos pacientes oncológicos, várias fórmulas de suplementos estão sendo lançadas no mercado, no intuito de diminuir a perda de peso desses pacientes e/ou diminuir o índice de complicações do tratamento (ORTEGA TT, 2016). Nesse sentido,os suplementos utilizados na atualidade são fórmulas enterais, imunomoduladoras fabricadas a base de arginina, glutamina, ômega 3 , nucleotídeos e outros componentes. Ademais, estes compostos geram impactos positivos na recuperação de pacientes com cânceres de cabeça e pescoço (KOWATA CH, et al., 2009).

De acordo com Vale IAV, et al. (2015), como a dieta imunomoduladora contém diversos componentes, é difícil atribuir efeitos da fórmula a um componente específico, sendonaturala necessidade de compreender qual o papel de cada suplemento nesses tipos de dietas.

Segundo Barbosa LBG, et al. (2017), o aminoácido arginina é caracterizado como semi-essencial ou parcialmente essencial ao organismo humano, uma vez que pode ser produzida endogenamente em uma quantidade necessária para entender as necessidades corporais. Todavia, em situações específicas como em casos de câncer, a concentração da arginina torna-se deficiente, bem como a diminuição da sua efetividade na atividade metabólica, sendo necessária a complementação através de sua ingestão.

Além disso, este aminoácido atua na estimulação da secreção do hormônio do crescimento, o qual tem por função acelerar os processos de cicatrização, como também inibir a perda de massa muscular, o que é entendido como de fundamental importância para pacientes oncológicos com déficits energéticos (VAN BDE, et at., 2001). De acordo com Novaes MRCG, et al. (2004), em relação ao tratamento de pacientes com câncer de cabeça e pescoço, a arginina vem sendo muito utilizada na suplementação desses pacientes na perspectiva de melhorar as condições, estímulos e ações do sistema imunológico, resultando, assim, em aumento da produção de Linfócitos T, citocinas, interleucinas e óxido nítrico contra a neoplasia diminuindo as altas incidências de infecção.

Nesse sentido, diante do apresentado, é de fundamental importância fazer uma revisão sistemática da literatura sobre os efeitos da suplementação com arginina em pacientes com câncer de cabeça e pescoço, além de verificar quais os efeitos benéficos deste suplemento em pacientes oncológicos de cabeça e pescoço e o impacto nas complicações cirúrgicas.Portanto, este trabalho tem como objetivo revisar o uso especificamente da arginina, que é componente presente nas dietas imunomoduladoras. 


\section{MÉTODOS}

Foi realizada uma revisão sistemática da literatura utilizando a estratégia PICO [Paciente ou Problema, Intervenção, Comparação, Outcomes (resultados)] (SANTOS CM, 2007)com o objetivo de responder a seguinte questão:Quais os impactos da suplementação com arginina em pacientes com câncer de cabeça e pescoço submetidos a tratamento cirúrgico?

Os critérios de inclusão foram: ensaios clínicos randomizados, desde que o idioma estivesse em inglês, espanhol ou português, sem qualquer restrição quanto a data de publicação, que avaliaram o efeito da suplementação do aminoácido arginina em pacientes com câncer de cabeça e pescoço em tratamento. Foram excluídos estudos que envolveram outros tipos de câncer, artigos que não abordaram o tema nutricional como foco da análise (discorreram acerca de outros processos do câncer em que a arginina se torna presente) e estudos com animais ou crianças.

A seleção dos artigos foi realizada por dois revisores independentes, sendo revistas por um terceiro revisor no período de julho a agosto de 2018. A busca foi realizada nas bases de dados PubMed, CENTRAL e Lilacs, utilizando as palavras-chave: "Head and neck neoplasms" AND "arginine" nas duas primeiras bases supracitadas e "Neoplasias de cabeça e pescoço" AND "Arginina" na última. Utilizamos os descritores Medical Subject Heading (MeSH) e Descritores em Ciências da Saúde (DeCS) para determinar os termos utilizados na pesquisa.

\section{RESULTADOS E DISCUSSÃO}

Foram encontrados 108 artigos nas bases PubMed, CENTRAL e Lilacs. Destes, 96 foram eliminados por se tratar de estudos que não abordaram o tema, que eram artigos repetidos ou ainda por não atenderem ao objetivo do presente estudo (Figura 1). Assim, chegamos a um total de 12 artigos, os quais foram analisados na íntegra e utilizados nesta revisão.

Os artigos incluídos foram avaliados de forma cega e independente por dois revisores, que selecionaram os estudos relevantes e aplicaram os critérios de elegibilidade; em caso de discordância, um terceiro revisor foi solicitado. Posteriormente, os estudos foram avaliados de forma integral com aplicação dos critérios de elegibilidade. E por fim, foram definidos os estudos incluídos e excluídos da revisão sistemática.

Figura 1 - Fluxograma de seleção dos artigos.

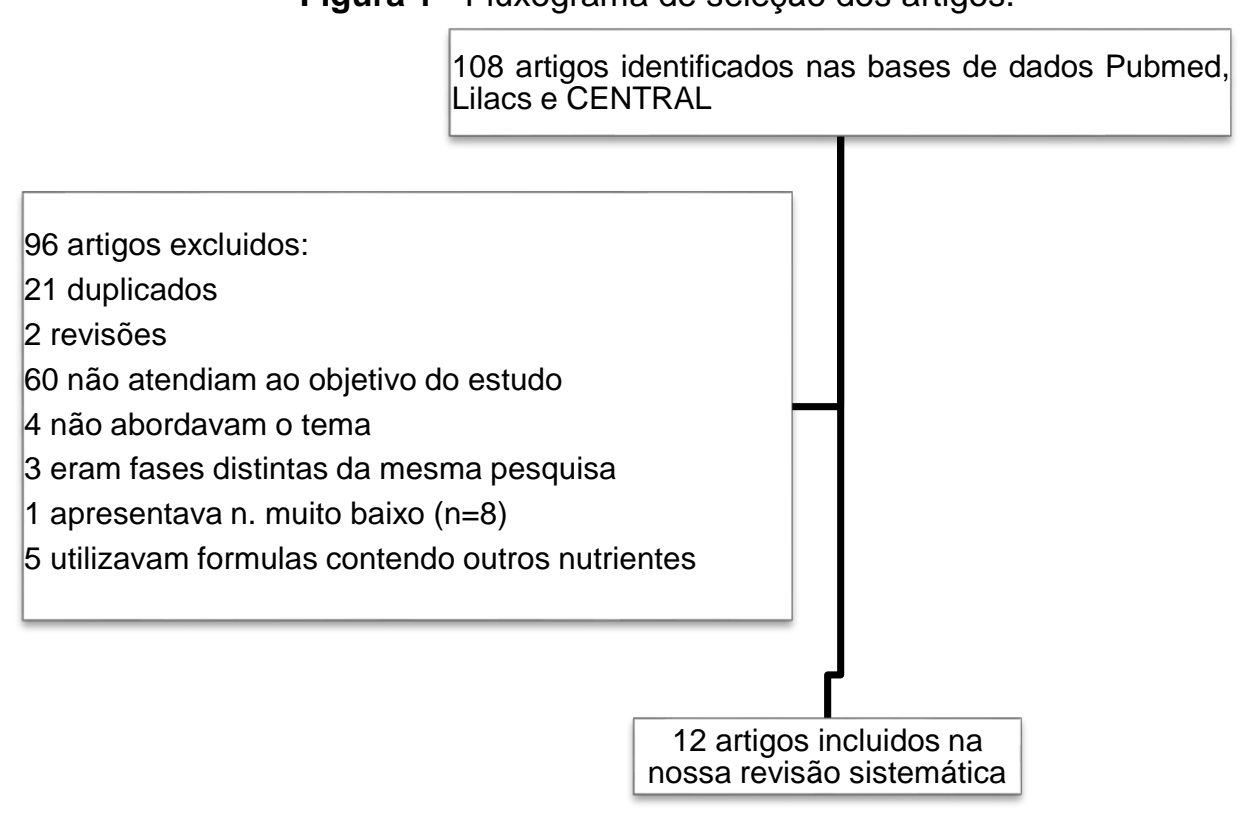

Fonte: Dados da pesquisa, 2018. 
Devido à heterogeneidade dos estudos, os dados foram agrupados e analisados por desfechos de modo descritivo. Dos 12 trabalhos encontrados, 9 compararam a suplementação de arginina com a dieta padrão enteral pós-operatória (Tabela 1).

Tabela 1 - Trabalhos comparando a suplementação com arginina versus dieta padrão em pós-operatório de ressecções de tumores de cabeça e pescoço.

\begin{tabular}{|c|c|c|c|}
\hline Autor/ano & População & Metodologia & Resultados \\
\hline $\begin{array}{l}\text { LUIS DA, et } \\
\text { al., } 2009\end{array}$ & $\begin{array}{l}72 \text { pacientes em } \\
\text { pós-operatório (PO) } \\
\text { de ressecções de } \\
\text { câncer de boca e } \\
\text { laringe }\end{array}$ & $\begin{array}{l}\text { Pacientes divididos no pós-operatório em } 2 \text { grupos: } \\
\text { grupo I recebeu fórmula enriquecida com arginina, grupo } \\
\text { II de controle recebeu fórmula enteral isocalórica e } \\
\text { isoprotéica. }\end{array}$ & $\begin{array}{l}\text { Diminuição do índice de fístulas no PO no grupo da } \\
\text { arginina }\end{array}$ \\
\hline $\begin{array}{l}\text { LUIS DA, et } \\
\text { al., } 2009\end{array}$ & $\begin{array}{l}41 \text { pacientes com } \\
\text { câncer de cabeça e } \\
\text { pescoço, com uma } \\
\text { perda de peso de } \\
5 \%-10 \% \text { nos } 3 \\
\text { meses anteriores } \\
\text { que roram } \\
\text { submetidos a } \\
\text { ressecções da } \\
\text { cabeça e pescoço }\end{array}$ & $\begin{array}{l}\text { Pacientes divididos no pós-operatório em } 2 \text { grupos: } \\
\text { grupo I recebeu fórmula enriquecida em arginina durante } \\
21 \text { dias, enquanto no grupo II os pacientes receberão } \\
\text { uma fórmula isocalórica e isoprotéica. }\end{array}$ & $\begin{array}{l}\text { Não houve diferenças entre os dois grupos em relação } \\
\text { aos níveis dos marcadores inflamatórios. }\end{array}$ \\
\hline $\begin{array}{l}\text { VAN BDE, et } \\
\text { at., } 2001\end{array}$ & $\begin{array}{l}49 \text { pacientes com } \\
\text { câncer de cabeça e } \\
\text { pescoço } \\
\text { gravemente } \\
\text { desnutridos }\end{array}$ & $\begin{array}{l}\text { Os pacientes foram alocados em três grupos com } \\
\text { alimentação respectivamente: padrão c/ sonda no pós- } \\
\text { operatório; padrão com sonda no pré e pós-operatório; e } \\
\text { suplementada c/ arginina no pré e pós-operatório. }\end{array}$ & $\begin{array}{l}\text { Foi observada uma tendência para uma melhor } \\
\text { sobrevivência no grupo suplementado com arginina }\end{array}$ \\
\hline $\begin{array}{l}\text { LUIS DA, et } \\
\text { al., } 2009\end{array}$ & $\begin{array}{l}90 \text { pacientes com } \\
\text { câncer de boca e de } \\
\text { laringe }\end{array}$ & $\begin{array}{l}\text { Na cirurgia, os pacientes foram alocados aleatoriamente } \\
\text { em dois grupos: grupo I pacientes que receberam um } \\
\text { suplemento de dieta enteral com arginina e grupo II com } \\
\text { pacientes que receberam uma fórmula isocalórica, } \\
\text { isoprotéica }\end{array}$ & $\begin{array}{l}\text { A tolerância gastrointestinal foi melhor no grupo II do que } \\
\text { em I ( } 40 \% \text { grupo I e } 13 \% \text { grupo II). As complicações pós- } \\
\text { operatórias devido a infecções foram semelhantes nos } \\
\text { dois grupos ( } 4 \% \text { grupo I e } 9 \% \text { grupo II). Fístula foi menos } \\
\text { freqüente no grupo nutricional enriquecido ( } 5 \% \text { grupo I e } \\
11 \% \text { grupo II); }\end{array}$ \\
\hline
\end{tabular}

REAS/EJCH |Vol. Sup.27 | e760 | DOI: https://doi.org/10.25248/reas.e760.2019 Página 4 de 8 


\begin{tabular}{|c|c|c|c|}
\hline $\begin{array}{l}\text { NIKKI BUJS, } \\
2010 .\end{array}$ & $\begin{array}{l}32 \text { pacientes } \\
\text { gravemente } \\
\text { desnutridos com } \\
\text { câncer de cabeça e } \\
\text { pescoço. }\end{array}$ & $\begin{array}{l}\text { Foram divididos em dois grupos: grupo I recebeu } \\
\text { nutrição enteral perioperatória padrão (grupo controle). } \\
\text { grupo II recebeu suplementação entérica perioperatória } \\
\text { com arginina (grupo arginina. }\end{array}$ & $\begin{array}{l}\text { O grupo que recebeu nutrição enriquecida com arginina } \\
\text { apresentou uma sobrevida global significativamente } \\
\text { melhor e melhor sobrevida específica para a doença } \\
\text { Além disso, o grupo suplementado com arginina } \\
\text { apresentou uma sobrevida livre de recidiva locorregiona } \\
\text { significativamente maior. }\end{array}$ \\
\hline $\begin{array}{l}\text { ROMAN NR, } \\
\text { et al., } 2016 .\end{array}$ & $\begin{array}{l}195 \text { pacientes } \\
\text { cirúrgicos } \text { com } \\
\text { câncer de cabeça e } \\
\text { pescoço de alto } \\
\text { risco. }\end{array}$ & $\begin{array}{l}\text { Foi oferecido para todos os pacientes do grupo de } \\
\text { intervenção uma preparação pré-operatória por } 5 \text { dias de } \\
\text { uma fórmula nutricional a base de Arginina. Uma similar, } \\
\text { fórmula comercialmente foi administrada por sonda } \\
\text { baseada no peso corporal dos pacientes até pelo menos } \\
5 \text { dias pós-operatório. }\end{array}$ & $\begin{array}{l}\text { O grupo que recebeu dieta enriquecida com arginina teve } \\
\text { menor índice de complicações e de fístulas pós- } \\
\text { operatórias. }\end{array}$ \\
\hline $\begin{array}{l}\text { LUIS DA, et } \\
\text { al., } 2009\end{array}$ & $\begin{array}{l}29 \text { pacientes com } \\
\text { câncer de boca e } \\
\text { laringe foram } \\
\text { divididos em dois } \\
\text { grupos }\end{array}$ & $\begin{array}{l}\text { Os pacientes do grupo A receberam dieta entérica com } \\
\text { arginina (grupo } \mathrm{l}, \mathrm{n}=14 \text { ); (B) pacientes receberam } \\
\text { fórmula entérica isoprotéica isocalórica sem arginina. }\end{array}$ & $\begin{array}{l}\text { Não houve diferenças significativas entre grupos e nos } \\
\text { níveis de proteínas séricas. Houve melhora significativa } \\
\text { nos níveis de pré-albumina, transferrina. PCR e IL-6. Não } \\
\text { houve alteração nos níveis de TNF e contagem de } \\
\text { linfócitos. }\end{array}$ \\
\hline $\begin{array}{l}\text { LUIS DA, et } \\
\text { al., } 2009\end{array}$ & $\begin{array}{lr}\text { Estudou } & 72 \\
\text { pacientes uma } \\
\text { população de } 72 \\
\text { pacientes com } \\
\text { câncer de boca e } \\
\text { laringe foi inscrita. }\end{array}$ & $\begin{array}{l}\text { Foram divididos em dois grupos, grupo I pacientes } \\
\text { receberam suplementos de dieta enteral com arginina; } \\
\text { grupo II pacientes receberam uma fórmula enteral } \\
\text { isocalórica e isoprotéica. }\end{array}$ & $\begin{array}{l}\text { Fístula foi menos freqüente no grupo com suplementação } \\
\text { de arginina ( } 5,2 \% \text { grupo I e } 17,6 \% \text { grupo II). O tempo de } \\
\text { permanência no pós-operatório foi melhor } 24,3 \pm 14 \text { dias } \\
\text { no grupo de imunonutrição do que } 36,1 \pm 27 \text { dias no } \\
\text { grupo controle. }\end{array}$ \\
\hline $\begin{array}{l}\text { RISO S, et } \\
\text { al., } 2000 \text {. }\end{array}$ & 44 pacientes. & $\begin{array}{l}\text { Os pacientes foram divididos em dois grupos para } \\
\text { receber: grupo I dieta enteral enriquecida com arginina e } \\
\text { grupo II, dieta controlada isocalórica e isoprotéica. }\end{array}$ & $\begin{array}{l}\text { Não houve diferença intergrupos significativa na } \\
\text { tendência das proteínas plasmáticas }\end{array}$ \\
\hline
\end{tabular}

Fonte: Dados da pesquisa, 2018.

$\overline{\text { REAS/EJCH |Vol. Sup.27 | e760 | DOI: https://doi.org/10.25248/reas.e760.2019 Página } \mathbf{5} \text { de } \mathbf{8}}$ 
Dos estudos analisados, dois trabalhos fizeram a comparação da arginina dada em diferentes doses, como evidenciado na Tabela 2, sendo que o principal achado foi a diminuição na freqüência de formação de fístulas nos pacientes tratados com doses elevadas de nutrição enteral contendo o aminoácido arginina.

Tabela 2 - Trabalhos comparando a suplementação com arginina em diferentes doses.

\begin{tabular}{|c|c|c|c|}
\hline Autor/ano & População & Metodologia & Resultados \\
\hline $\begin{array}{l}\text { LUIS DA, et } \\
\text { al., } 2009\end{array}$ & $\begin{array}{l}84 \text { pacientes com } \\
\text { câncer de boca e } \\
\text { laringe }\end{array}$ & $\begin{array}{l}\text { Foram separados aleatoriamente os } \\
\text { pacientes em } 3 \text { grupos. A partir da } \\
\text { cirurgia cada grupo recebeu um suporte } \\
\text { nutricional entérico com dosagem } \\
\text { diferente, sendo, grupo I: } 5,7 \mathrm{~g} \text { de } \\
\text { arginina por dia (baixa dose); grupo II: } \\
12,3 \mathrm{~g} \text { de arginina por dia (dose média) } \\
\text { e grupo III, } 18,9 \mathrm{~g} \text { de arginina por dia } \\
\text { (dose elevada). }\end{array}$ & $\begin{array}{l}\text { Fístulas foram menos } \\
\text { frequentes nos grupos I e II do } \\
\text { que no grupo III ( } 3,6 \% \text { grupo I } \\
\text { vs 3,6\% grupo II vs } 10,7 \% \\
\text { grupo III) }\end{array}$ \\
\hline $\begin{array}{l}\text { LUIS DA, et } \\
\text { al., } 2009\end{array}$ & $\begin{array}{lr}\text { estudaram } & 115 \\
\text { pacientes. Sendo } \\
\text { distribuídos em } \\
\text { dois grupos: } \\
\text { grupo I } & (58 \\
\text { pacientes) } & \text { e } \\
\text { grupo II } & (57 \\
\text { pacientes) } & \end{array}$ & $\begin{array}{l}\text { grupo I recebeu dieta enteral com alta } \\
\text { dose de arginina ( } 20 \mathrm{~g} \text { por dia) e grupo } \\
\text { II (57 pacientes) receberam uma } \\
\text { fórmula enteral isocalórica e isoprotéica } \\
\text { com um meio de dose de arginina ( } 12,3 \\
\text { g por dia). }\end{array}$ & $\begin{array}{l}\text { As complicações das } \\
\text { infecções pós-operatórias } \\
\text { foram semelhantes nos dois } \\
\text { grupos ( } 8,6 \% \text { do grupo I e } \\
12,2 \% \text { do grupo II). A fístula foi } \\
\text { menos frequente no grupo } \\
\text { nutrição enriquecida ( } 3,4 \% \\
\text { grupo I e } 10,5 \% \text { grupo II }\end{array}$ \\
\hline
\end{tabular}

Fonte: Dados da pesquisa, 2018.

Um trabalho comparou a dieta composta de arginina com a dieta imunomoduladora, como descrito na Tabela 3, o qual demostrou que não houve melhora estatística das fístulas em pacientes que tiveram dieta aumentada, além de não ter ocorrido diferenças significativas quanto concentração e composição plasmática de proteínas e células de defesa e estruturas pró-inflamatórias.

Tabela 3 - Trabalho comparando a suplementação com arginina com a dieta padrão e dieta imunomoduladora.

\begin{tabular}{|c|c|c|c|}
\hline Autor/ano & População & Metodologia & Resultados \\
\hline $\begin{array}{l}\text { CASAS RP et } \\
\text { al., } 2007\end{array}$ & $\begin{array}{l}\text { Uma população de } 44 \\
\text { pacientes com câncer de } \\
\text { boca e laringe foi } \\
\text { recrutada em um estudo } \\
\text { randomizado. }\end{array}$ & $\begin{array}{l}\text { Sendo divididos em três grupo: } \\
\mathrm{n} 1=15, \mathrm{n} 2=15, \mathrm{n} 3=14 \text {. O grupo } \\
\text { I incluiu pacientes que } \\
\text { receberam uma dieta enteral } \\
\text { suplementada com arginina, o } \\
\text { grupo II incluiu pacientes que } \\
\text { receberam uma fórmula enteral } \\
\text { polimérica padrão, e o grupo III } \\
\text { incluiu pacientes que } \\
\text { receberam uma dieta enteral } \\
\text { suplementada com arginina, } \\
\text { ácido ribonucleico e ômega } 3 \text {. }\end{array}$ & $\begin{array}{l}\text { Infecções de feridas e } \\
\text { infecções gerais foram mais } \\
\text { frequentes no grupo controle. } \\
\text { As taxas de fístula não } \\
\text { melhoraram nos grupos de } \\
\text { dieta aumentada. Não foram } \\
\text { detectadas diferenças } \\
\text { intergrupos significativas na } \\
\text { tendência das duas proteínas } \\
\text { plasmáticas (albumina, } \\
\text { transferrina), linfócitos, peso, } \\
\text { IL-6, RCP e TNFa. }\end{array}$ \\
\hline
\end{tabular}

Fonte: Dados da pesquisa, 2018.

Ao analisarmos os trabalhos observamos que nenhum dos trabalhos que constatou um aumento das proteínas plasmáticas nos grupos com dieta suplementada com arginina (LUIS DA, et al., 2009; LUIS DA, et al., 2004; LUIS DA, et al., 2007; LUIS DA, et al., 2005; RISO S, 2000). 
O trabalho de Luis DA, et al. (2005) que compara a dieta rica em ômega 3 com a dieta rica em Arginina observou um aumento das proteínas plasmáticas no grupo do ômega 3. Não houve diferenças estatísticas nos níveis séricos dos marcadores inflamatórios nos trabalhos que avaliaram esta variável (LUIS DA, et al.,2007; LUIS DA, et al., 2009; LUIS DA, et al., 2004; LUIS DA, et al., 2005; LUIS DA, et al., 2009).

Os trabalhos de Luis DA, et al., (2007); Luis DA, et al., (2004); Rowan, et al., (2016) e o de Luis DA, et al. (2009) encontraram uma diminuição no índice de fístulas pós operatórias nos pacientes que fizeram uso da suplementação com arginina versus dieta padrão no pós operatório.Por outro lado o estudo de Casas-Rodera P (2007) não observou diferenças estatísticas no índice de fístulas pós operatória.

Os trabalhos de Luis DA, et al., (2004), Luis DA, (2015) e de Casas-Rodera P, (2007) não observaram diferenças nos índices de infecção PO. Observamos que os trabalhos são bem divergentes quanto a metodologia empregada alguns comparando a arginina com a dieta padrão, outros comparando arginina em diversas doses e outro com as dietas imunomoduladoras que já contém a arginina em sua fórmula. A dose utilizada da arginina também foi bem heterogênea $5,7 \mathrm{~g}$ a $20 \mathrm{~g}$ ao dia.

Porém a observamos um impacto muito grande da arginina na diminuição de fístulas no pós operatório nos trabalhos de Luis DA, et al., (2007); Luis DA, et al., (2004); Rowan, et al., (2016) e Luis DA, et al., (2009), que é uma complicação relativamente frequênte em cirurgias de grande porte na cabeça e pescoço. $O$ que representaria uma consequente diminuição do tempo de internação hospitalar e morbidade destes pacientes.

O nosso estudo tem duas principais limitações: a primeira é da heterogenicidade dos métodos empregados nos artigos escolhidos. E a segunda é do fato de que a própria dieta imunomoduladora já contém a arginina, sendo difícil atribuir um beneficio para um componente especifico da fórmula. Acreditamos que no futuro mais trabalhos sobre arginina e dietas imunomuduladoras sejam realizados e mais pacientes possam ter acesso a estes suplementos que como pudemos observar diminuem o índice de complicações cirúrgicas em especial de fístulas, melhorando a qualidade da assistência aos pacientes com câncer de cabeça e pescoço submetidos a cirurgia.

\section{CONSIDERAÇÕES FINAIS}

Baseado nos trabalhos avaliados, a suplementação com arginina em pacientes com câncer de cabeça e pescoço não constatou um aumento das proteínas plasmáticas. Não houve também diferenças estatísticas nos níveis séricos dos marcadores inflamatórios. Os índices de infecção PO também permaneceram sem diferenças estatísticas. Porém houve uma diminuição no índice de fístulas pós-operatórias nos pacientes que fizeram uso da suplementação com arginina versus dieta padrão no pós-operatório.

\section{AGRADECIMENTOS E FINANCIAMENTO}

Agradecemos ao Hospital de câncer de Barretos e em especial a direção do Hospital de Amor Amazônia pela oportunidade de desenvolver pesquisa e pelo apoio nas batalhas do dia a dia.

\section{REFERÊNCIAS}

1. AGGARWAL BB, et al. Inflammation and cancer: How friendly is the relationship for cancer patients? Curr Opin Pharmacol, 2009; Vol. 9: 351-369.

2. BARBOSA LBG, et al. Impacto de fórmulas enterais imunomoduladoras em pacientes com câncer do trato gastrointestinal enteral: uma revisão da literatura. J Health Sci Inst. Brasília, 2017, Vol. 35: 49-54.

3. CASAS RP, et al.Immune Enhancing enteral nutrition formulas in head and neck cancer surgery: a prospective, randomized clinical trial. Nutrición Hospitalaria, 2007, Vol. 28: 105-110.

4. LUIS DA, et al. A Randomized Clinical Trial with Oral Immunonutrition (w3-Enhanced Formula vs. Arginine-Enhanced Formula) in Ambulatory Head and Neck Cancer Patients. Annals of Nutrition Metabolism, 2005, Vol. 49: 95-99.

5. LUIS DA, et al. A randomized double-blind clinical trial with two different doses of arginine enhanced enteral nutrition in postsurgical cancer patients. European Journal of Clinical Nutrition, 2010, Vol. 14: 941-945. 
6. LUIS DA, et al. Clinical and biochemical outcomes after a randomized trial with a high dose of enteral arginine formula in postsurgical head and neck câncer patients. European Journal of Clinical Nutrition, 2007, Vol. 16: 200-204.

7. LUIS DA, et al. Efecto de una fórmula enteral enriquecida en arginina sobre los marcadores inflamatorios en pacientes con tumores de cabeza y cuello. Med Clin, 2009, Vol. 132, 2 : 49-52.

8. LUIS DA, et al. High dose of arginine enhanced enteral nutrition in postsurgical head and neck cancer patients. A randomized clinical trial. European Journal of Clinical Nutrition, 2009: 279-283.

9. LUIS DA, et al.Randomized clinical trial with an enteral arginine-enhanced formula in early postsurgical head and neck cancer patients. European Journal of Clinical Nutrition, 2004, Vol. 58: 505-1508.

10. LUIS DA, et al. Effect of three different doses of arginine enhanced enteral nutrition on nutritional status and outcomes in well nourished postsurgical cancer patients: a randomized single blinded prospective trial. Eur Ver Med Pharmacol, 2015, Vol. 19: 950-955.

11. FRUCHTEBICHT AVG, et al. Inflammatory and nutritional statuses of patients submitted to resection of gastrointestinal tumor. Rev Col Bras Cir., 2018., Vols. 45, n.2: 1-11.

12. GALBIATTI ALS et al. Câncer de cabeça e pescoço: causas, prevenção e tratamento. Braz. j. otorhinolaryngol. [online], 2013, vol.79, n.2: 239-247.

13. INCA, [ed.]. Estimativa 2018: incidência de câncer no Brasil / Instituto Nacional de Câncer José Alencar Gomes da Silva - Rio de Janeiro: INCA, 2017.

14. KOWATA CH et al. Fisiopatologia da caquexia no câncer: uma revisão. Arq. Ciênc. Saúde UNIPAR, Umuarama, 2009. v. 13, n. 3, p. 267-272.

15. NIKKI BUJS, et al. Perioperative arginine-supplemented nutrition in malnourished patientswith head and neck cancer improves long-term survival. Am J Clin Nutr, 2010, Vol. 92: 1151-6.

16. NOVAES MRCG, et al. Farmacologia da L-arginina em pacientes com câncer. Revista Brasileira de Cancerologia, 2004, Vol. 50, 4: 321-325.

17. ORTEGA TT. O Impacto do uso de imunonutrientes na terapia. Rev. Bras. Cir. Cabeça Pescoço, 2016: $154-158$.

18. RISO S, et al. Postoperative enteral immunonutrition in head and neck cancer patients. Clinical Nutrition, 2000, Vol. 19, pp. 407-412.

19. ROWAN, N.R., et al. Utility of a perioperative nutritional intervention on postoperative outcomes in high risk head and neck cancer patients. Oral Oncology, 2016, Vol. 54: 42-46.

20. SANTOS CM. The PICO strategy for the research question construction and evidence search. . Rev. Latino-am. Enfermagem, 2007., Vol. 15, 3: 508-5011.

21. SILVA MPN, et al. Síndrome da anorexia-caquexia em portadores de câncer. Revista Brasileira de Cancerologia, 2006; 52(1): 59-77

22. VALE IAV, et al. Nutritional Assessment and Recommendation in Cancer Patients Initiating Chemotherapy. Revista Brasileira de Cancerologia, 2015; 61(4): 367-372.

23. VAN BMA, et al. Effect of perioperative nutrition, with and without arginine supplementation, on nutritional status, immune function, postoperative morbidity, and survival in severely malnourished head and neck cancer patients. Am. J. Clin. Nutr., 2001, Vol. 73: 323-332. 